\section{PSICOLOGÍA IBEROAMERICANA}

\section{Psicología lberoamericana}

ISSN: 1405-0943

psicología.iberoamericana@uia.mx

Universidad Iberoamericana, Ciudad de

México

México

Sánchez Martínez, Cynthia

Significado psicológico de familia, papá y mamá en adolescentes

Psicología Iberoamericana, vol. 20, núm. 1, enero-junio, 2012, pp. 18-28

Universidad Iberoamericana, Ciudad de México

Distrito Federal, México

Disponible en: http://www.redalyc.org/articulo.oa?id=133924623003

- Cómo citar el artículo

- Número completo

- Más información del artículo

Página de la revista en redalyc.org

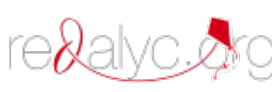

Sistema de Información Científica

Red de Revistas Científicas de América Latina, el Caribe, España y Portugal

Proyecto académico sin fines de lucro, desarrollado bajo la iniciativa de acceso abierto 


\title{
Significado psicológico de familia, papá y mamá en adolescentes
}

\author{
Psychological Meaning of Family, Father and Mother in Adolescents
}

\author{
Cynthia Sánchez Martínez \\ FACULTAD de Psicología \\ Universidad Michoacana de San Nicolás de Hidalgo, México*
}

\section{RESUMEN}

La siguiente investigación analiza los significados psicológicos que los adolescentes otorgan a la familia, con el objetivo de examinar las diferencias que se presentan a lo largo de las subetapas de la adolescencia. Se llevó a cabo un estudio no experimental en el que participaron 435 adolescentes de edades entre los nueve y 25 años, utilizando la técnica de Redes semánticas naturales (Valdez, 1991). Se observaron diferencias en cuanto a los significados psicológicos otorgados a dichas palabras en las distintas etapas de la adolescencia. Los hallazgos sugieren que en los estadios iniciales de ésta, los significados psicológicos están asociados a los elementos que integran la familia, mientras que los significados de los adolescentes de mayor edad se asocian a valores y emociones.

Descriptores: adolescencia, familia, mamá, papá, significado psicológico.

\section{ABSTRACT}

The present investigation analyzes the psychological meanings that adolescents give to the family, with the purpose of examining the differences that occur along the substages of adolescence. Was conducted a non experimental study, which involved 435 pre-adolescents and adolescents aged between nine and 25, using the technique of Natural Semantic Networks (Valdez, 1991), noticing significant differences in the psychological meanings given in the different stages of adolescence. Overall, the findings suggest that in the early stages of adolescence, the psychological meanings are less entrenched than the meanings of older adolescents.

Keywords: adolescence, family, mother, father, psychological meaning.

\section{INTRODUCCIÓN}

La familia, así como su concepto, ha sufrido transformaciones a lo largo de la historia en un intento por aproximarse a la comprensión del funcionamiento de las relaciones humanas. Estos cambios han sido objeto de estudio de diferentes disciplinas, de las cuales no es una excepción la psicología.

A lo largo de la revisión de la literatura se han elaborado múltiples definiciones de familia como una forma de contextualizar las implicaciones que ésta con- lleva. Al respecto Burgess y Locke (citado en Roche, 2006, p. 10), la definen como "una unidad de personas en interacción, relacionadas por vínculos de matrimonio, nacimiento o adopción cuyo objetivo central es crear y mantener una cultura común que promueva el desarrollo físico, mental, emocional y social de cada uno de sus miembros". En una definición menos tradicional, Estrada (2007) la describe como una célula social, cuya membrana protege en el interior a sus in-

\footnotetext{
* Para correspondencia: Cynthia Sánchez Martínez. Universidad Michoacana de San Nicolás de Hidalgo. Francisco Villa 450, col. Miguel Silva, C.P. 58110, Tel: 44331299 09, ext. 104, e-mail:cyn-colibri@live.com.mx
} 
dividuos y los relaciona al exterior con otros organismos semejantes.

De la misma forma, Gil (2007) señala que la familia debe asegurar la creación de vínculos afectivos, que funjan como precursores de otros; de manera que la unidad familiar otorga la fuerza y sentido a sus miembros desde su interior, pero que a la vez los relaciona y pone en contacto con el exterior.

Pero ¿qué significado tiene para los adolescentes la familia?, y ¿qué significado tienen papá y mamá? De acuerdo con diversas teorías cognitivas, los seres humanos tienden a cambiar su forma de pensamiento de acuerdo con la grado de madurez que adquieren a través de los años, siendo capaces de elaborar conceptos cada vez más complejos (Inhelder \& Piaget, 1996). Desde una perspectiva psicológica es necesario tomar en cuenta el mundo de significados ligado a la experiencia de cada sujeto, así como su edad y nivel de maduración. Bajo esta visión, es posible entonces pensar que el sentido y significado que los adolescentes tienen de los conceptos afines a la familia, tales como padre y madre, sufren un cambio importante de acuerdo con la fase de la adolescencia en que se encuentren.

La adolescencia es una etapa compleja del desarrollo, en la que la tarea esencial es la consolidación de la identidad, lo que conlleva una cierta vulnerabilidad para la aparición de conflictos entre padres e hijos adolescentes (Santrock, 2004). Dichos conflictos pueden ser explicados por los cambios en las estructuras cognitivas y emocionales a los que los jóvenes se ven sometidos, ya que en este camino hacia la consolidación de su identidad pasan por diversos estadios, en los cuales se permiten experimentar nuevas visiones de la realidad, que en muchas ocasiones parecen no coincidir con las de los padres.

Ante ello, conocer lo que los adolescentes sienten y piensan con respecto a sus familias es parte importante de la comprensión de dicha etapa, y un gran paso para que la familia atraviese esta fase de forma menos tumultuosa, a través de una visión más amplia desde las propias palabras del adolescente.

Así pues, la construcción del conocimiento científico en el campo de la psicología requiere de investigaciones que sustenten los cambios que se producen en el ser humano, por ejemplo los de la transición adolescente de la que se ha hablado; sin embargo, para que ello sea posible, es necesaria la elaboración y aplicación de nuevos instrumentos que permitan conocer y evaluar los significados psicológicos que los adolescentes otorgan a la familia, así como los elementos que se asocian a ella, o sea, el papá y la mamá, ya que cada uno de ellos es parte central de la misma.

De este modo, considerando la posición que ocupa la familia en la sociedad como pilar fundamental en la formación de individuos que hagan frente a los desafíos que implican subsistir en la compleja red de sistemas y organizaciones del mundo actual, la presente investigación aborda aspectos sobresalientes del sentido y significado que ésta tiene en los adolescentes, a partir de una técnica que permite obtener un reflejo claro de la visión que predomina en esta etapa de la vida, con lo que se busca actualizar las bases teóricas y profundizar sobre las características particulares que se otorgan al contexto familiar en las diferentes subetapas de la adolescencia. El propósito es identificar los cambios que suceden en el pensamiento adolescente en sus diferentes momentos, ya que ha de entenderse que las necesidades que ocupan a esta población varían considerablemente de las de los adultos.

Ante la escasez de instrumentos o métodos que definan el significado psicológico, los investigadores del campo de la psicología cognitiva continúan desarrollando técnicas que permitan la comprensión del comportamiento humano. Desde este campo, se explica que la conducta de los hombres se origina debido a los procesos de construcción y reconstrucción de la información que se elaboran a nivel mental. A partir de este fundamento, el desarrollo de la técnica de Redes naturales se vislumbra con un método confiable para evaluar el significado de palabras, ayudando a comprender la estructura de la memoria y la forma en que se asocian los significados (Zermeño, Arellano \& Ramírez, 2005).

En este sentido, como técnica de evaluación, las redes semánticas naturales permiten obtener datos que están altamente relacionados con la palabra estímulo, ya que los sujetos tienen total libertad para generar las acepciones que en su caso mejor definan el concepto central, sin verse limitados a tener que elegir sólo entre determinadas opciones.

Es así que en el presente estudio se planteó como objetivo conocer el significado psicológico que los 
adolescentes atribuyen a los conceptos de familia, papá y mamá, para con ello poder identificar en qué sentido se producen los cambios, bajo la hipótesis de que existen diferencias en dichos significados, de acuerdo con la fase en que se encuentren los adolescentes.

\section{MÉTODO}

La aplicación de la técnica de Redes semánticas naturales comprende una metodología cuanticualitativa. En su fase cuantitativa se realiza la obtención de palabras definidoras, para determinar el significado psicológico de los estímulos estudiados, en tanto que en su fase cualitativa se determina la distancia semántica cuantitativa de las palabras que conforman el núcleo de la red.

\section{Participantes}

Los participantes se eligieron través de un muestreo no probabilístico intencional, donde se obtuvo la participación de 435 adolescentes hombres y mujeres de diversas secundarias de la zona urbana de la ciudad de Morelia. Se conformaron cinco grupos de adolescentes de acuerdo con la correspondencia entre edad y subetapa de la adolescencia. El resultado fue $18.4 \%$ de preadolescentes de entre nueve y 11 años de edad, $20.7 \%$ de adolescentes tempranos de entre 12 y 15 años, $18.4 \%$ de adolescentes medios de entre 16 y 18 años, $28.7 \%$ de adolescentes tardíos de entre 19 y 21 años, y $13.8 \%$ de postadolescentes de entre 22 y 25 años.

\section{Instrumento}

Se utilizó la técnica de Redes semánticas naturales para conocer el significado psicológico de elementos de la familia en adolescentes, en las diferentes subetapas de la adolescencia. La red semántica natural de un concepto es aquel conjunto de conceptos elegidos por la memoria a través de un proceso reconstructivo, así como la evaluación subjetiva de los eventos acciones u objetos (Valdez, 2002).

\section{Procedimiento}

Se elaboró la ficha de Redes semánticas naturales, la cual contenía conceptos asociados a la familia, así como dos estímulos distractores. El instrumento estuvo conformado por cinco hojas tamaño esquela, con las palabras estímulo ubicadas en la parte superior. La primera hoja contenía las instrucciones y los datos ge- nerales de los participantes, así como la primer palabra estímulo: familia. De manera subsecuente, en las hojas dos y cuatro se colocaron las palabras estímulo: papá y mamá; como distractores se utilizaron las palabras amistad y noviazgo, en las hojas tres y cinco.

La técnica consistió en pedir a los participantes que definieran los estímulos familia, papá y mamá por medio de sustantivos, adjetivos y adverbios, y que jerarquizaran las definidoras en orden de importancia. La aplicación del instrumento se realizó de manera colectiva, en grupos de 15 a 30 participantes, en diversas escuelas de la ciudad de Morelia. El tiempo promedio de aplicación fue de 15 minutos.

Los datos obtenidos a partir de la técnica fueron vaciados al programa Microsoft Office Excel 2007; se ordenaron alfabéticamente y fueron agrupados de manera tal que se obtuvo el total de palabras definidoras o tamaño de red (TR), determinando el peso semántico (Ps) de las frecuencias numéricas ponderadas para cada palabra. Se calculó la distancia semántica cuantitativa (DSC) o valor FMG para cada definidora, asignando un valor del $100 \%$ a la palabra que más se acercó a cada uno de los estímulos.

\section{RESULTADOS}

\section{Preadolescencia}

Con base en los resultados obtenidos a través de la técnica de Redes semánticas naturales aplicada al grupo de preadolescentes, se obtuvo en primer lugar, el valor J o TR (tamaño de red) para cada palabra estímulo, lo cual, de acuerdo con Reyes Lagunes (1993), brinda un panorama general con respecto a la amplitud semántica generada por los sujetos, misma que estuvo conformada de la siguiente manera:

$$
\text { Familia }=184 \quad \text { Papá }=208 \quad \text { Mamá }=185
$$

Para determinar el conjunto SAM se tomaron las $10 \mathrm{pa}-$ labras con mayor valor $\mathrm{M}$, tal como lo proponen los iniciadores de la técnica, de tal forma que en las figuras $1,1.1$ y 1.2 se muestran los conjuntos para cada palabra estímulo, así como su valor FMG, el cual indica en términos cuantitativos la distancia semántica entre los elementos que componen el núcleo de la red. 
En el conjunto SAM del grupo de preadolescentes se observa que el nodo central del estímulo familia es mamá, con una distancia semántica de 100\% (valor FMG); también aparecen los términos papá con $91 \%$ y hermanos con $46 \%$.

Respecto al estímulo papá, la palabra trabajador aparece como nodo central (100\%), seguido de enojón (70\%) y bueno (69\%). Para el estímulo mamá, la palabra que funge como nodo central es bonita con $100 \%$ del valor FMG, cariñosa con $90 \%$ y buena $89 \%$.

Figura 1. Distancia semántica cuantitativa del estímulo familia en la preadolescencia

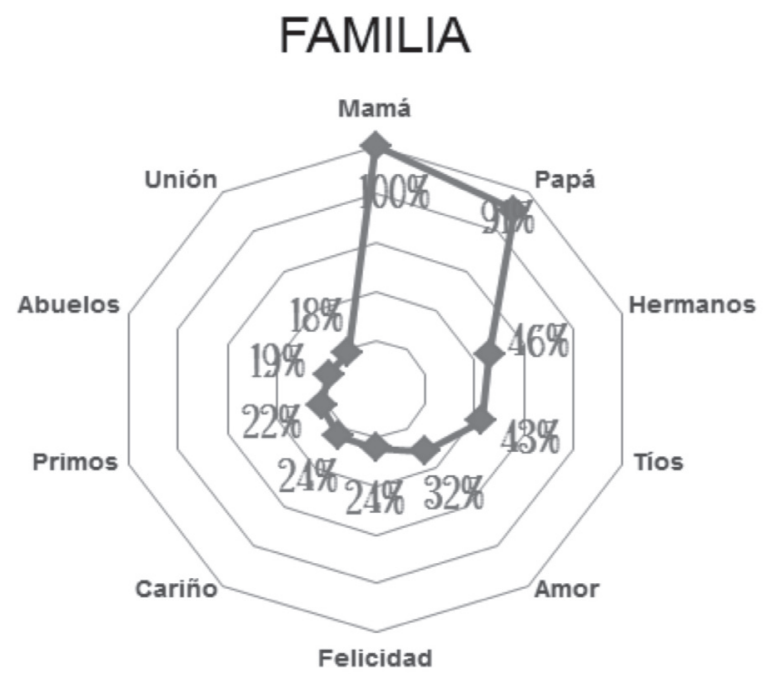

Figura 1.1. Distancia semántica cuantitativa del estímulo papá en la preadolescencia

\section{PAPÁ}

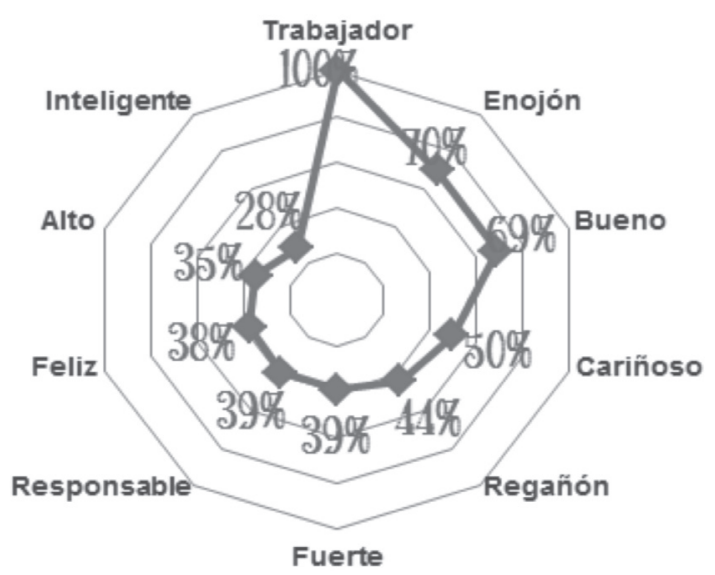

Figura 1.2. Distancia semántica cuantitativa del estímulo mamá en la preadolescencia

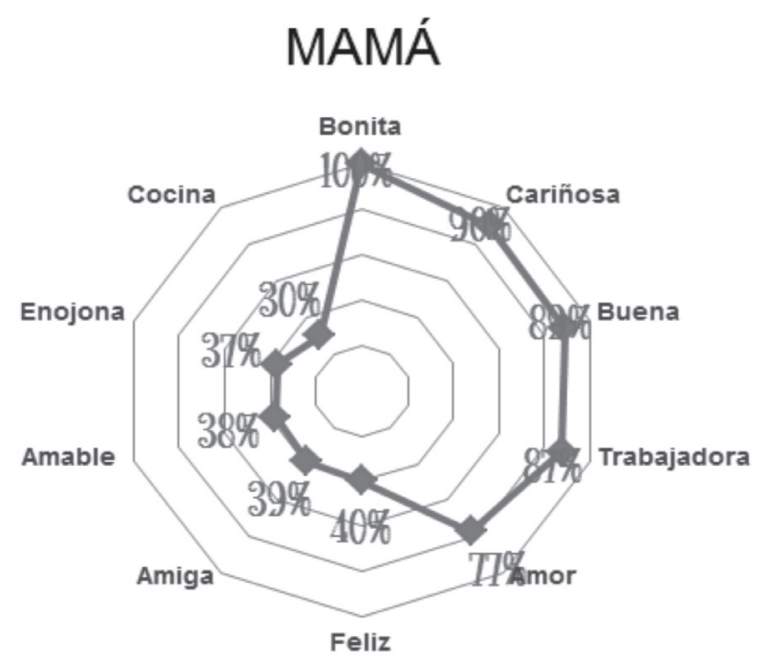




\section{Adolescencia temprana}

Para el grupo de adolescentes tempranos el tamaño de red o valor J para cada una de las palabras estímulo estuvo conformado de la siguiente manera:

$$
\text { Familia }=151 \quad \text { Papá=225 Mamá=218 }
$$

De la misma forma, el conjunto SAM y valor FMG se presentan en las gráficas 2, 2.1 y 2.2, mostrando la distancia semántica existente entre las palabras definidoras.

Figura 2. Distancia semántica cuantitativa del estímulo familia en la adolescencia temprana

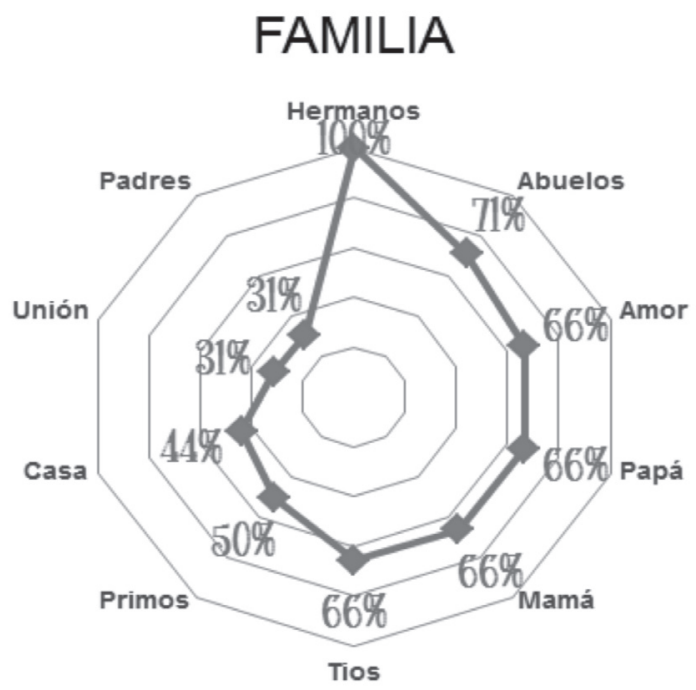

Figura 2.1. Distancia semántica cuantitativa del estímulo papá en la adolescencia temprana

\section{PAPÁ}

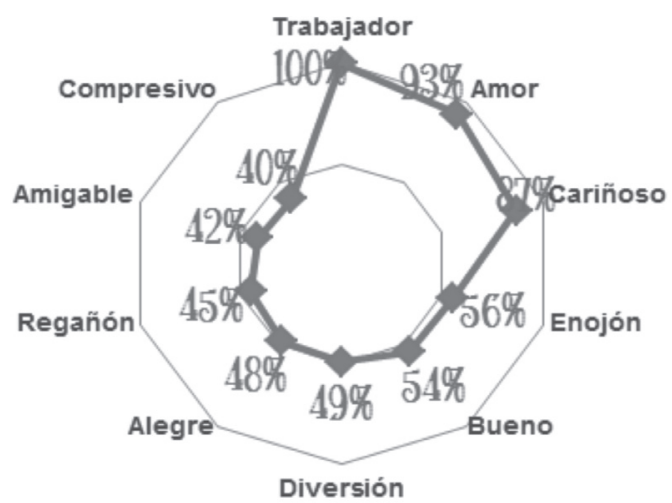

Figura 2.2. Distancia semántica cuantitativa del estímulo mamá en la adolescencia temprana

\section{MAMÁ}

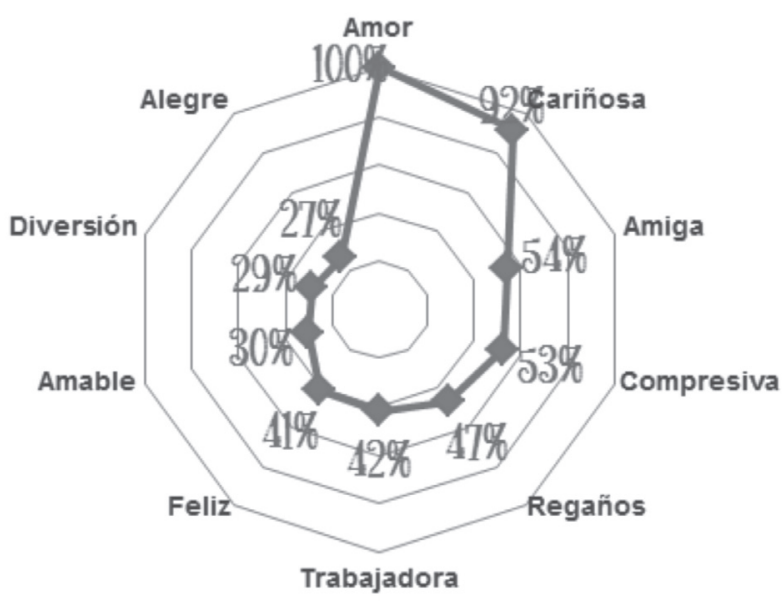

En las figuras anteriores es posible observar que en el estímulo familia, la definidora hermanos ocupa el primer lugar en el núcleo de la red $(\mathrm{FMG}=100 \%)$, seguida de abuelos (FMG $=71 \%)$. Hay cuatro definidoras que ocupan el tercer lugar en la red, y comparten el mismo valor FMG: amor, papá, mamá y tíos (FMG=66\%). Para el estímulo papá aparecen las definidoras: trabajador (100\%), amor (93\%) y cariñoso (87\%); en tanto que para mamá surgen las palabras amor (100\%), cariñosa (92\%) y amiga (54\%). 


\section{Adolescencia media o propiamente dicha}

El valor J del grupo de adolescentes cuya edad oscila entre los 16 y los 18 años aproximadamente; es decir, quienes se encuentran en la adolescencia media, estuvo conformado de la siguiente manera:

$$
\text { Familia }=106 \quad \text { Papá }=180 \quad \text { Mamá=155 }
$$

De este modo, las 10 definidoras que tuvieron los valores $\mathrm{M}$ más altos en cada una de las palabras estímulo, conformaron el conjunto SAM para este grupo de adolescentes, determinando así su valor FMG. La palabra amor resultó ser el nodo central (100\%) para el estímulo familia, seguido de unión con $51 \%$ y apoyo con $41 \%$.

Para el estímulo papá, se ubicó la palabra amor con $100 \%$ del valor FMG, respetuoso con $70 \%$ y trabajador con $54 \%$. En cuanto al estímulo mamá, en primer lugar se ubicó la definidora amor con el 100\%, seguido de cariñosa y apoyo con $41 \%$ y $28 \%$ respectivamente, tal como se muestra en las figuras $3,3.1$ y 3.2.

Figura 3. Distancia semántica cuantitativa del estímulo familia en la adolescencia media

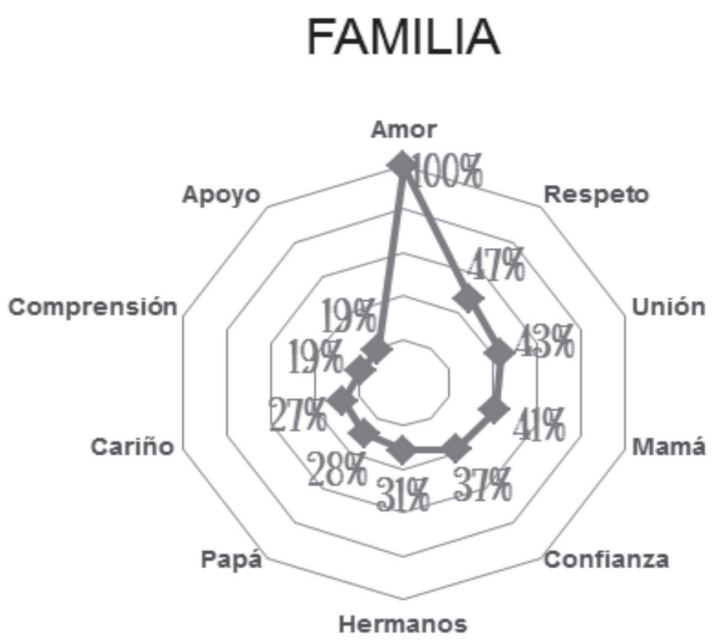

Figura 3.1. Distancia semántica cuantitativa del estímulo papá en la adolescencia media

\section{MAMÁ}

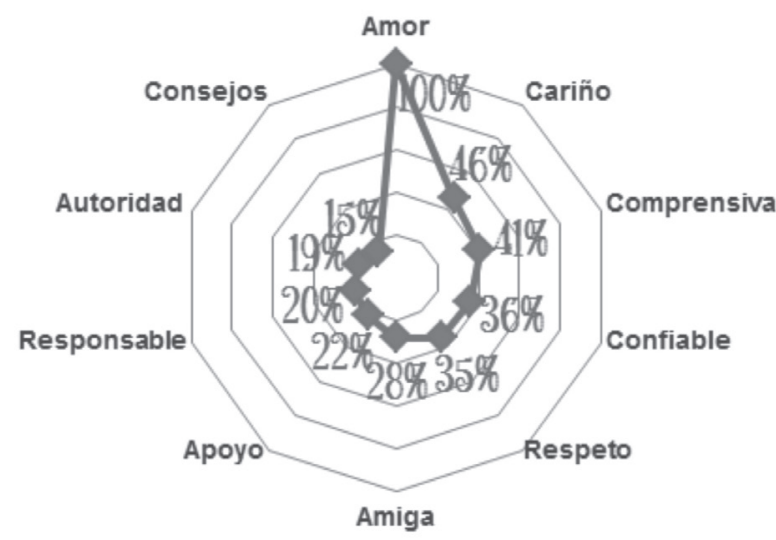

Figura 3.2. Distancia semántica cuantitativa del estímulo mamá en la adolescencia media

\section{PAPÁ}

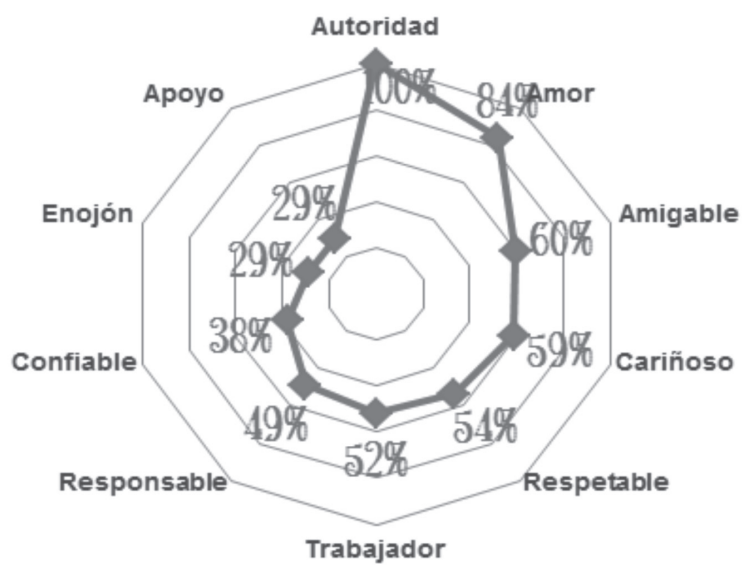




\section{Adolescencia tardía}

El valor J de los estímulos explorados en la adolescencia tardía se distribuyó de la siguiente manera:

\section{Familia $=123 \quad$ Papá $=189 \quad$ Mamá=158}

En relación con el conjunto SAM y valor FMG para el estímulo familia, se observa que el concepto central de la red semántica es amor, con una distancia de $100 \%$, unido con una distancia de $65 \%$, seguido de madre y confiable (ambas con $49 \%$ del valor FMG).

En relación con el estímulo papá, con la palabra autoridad fue el núcleo central de la red ( $\mathrm{FMG}=100 \%)$; el segundo lugar lo ocupó respeto (88\%), y el tercero responsable, con un $78 \%$ del valor FMG.

De la misma forma que para el estímulo familia, el nodo central de mamá, fue la palabra amor con un valor FMG de $100 \%$, aparecen también las palabras confiable y respeto, con un 35\% y $33 \%$ respectivamente.

De esta manera, el conjunto SAM, así como su valor FMG para cada palabra estímulo, puede ser observado en la figura 4 para el estímulo familia, 4.1 para papá y finalmente, en la figura 4.2 para mamá.

Figura 4. Distancia semántica cuantitativa del estímulo familia en la adolescencia tardía

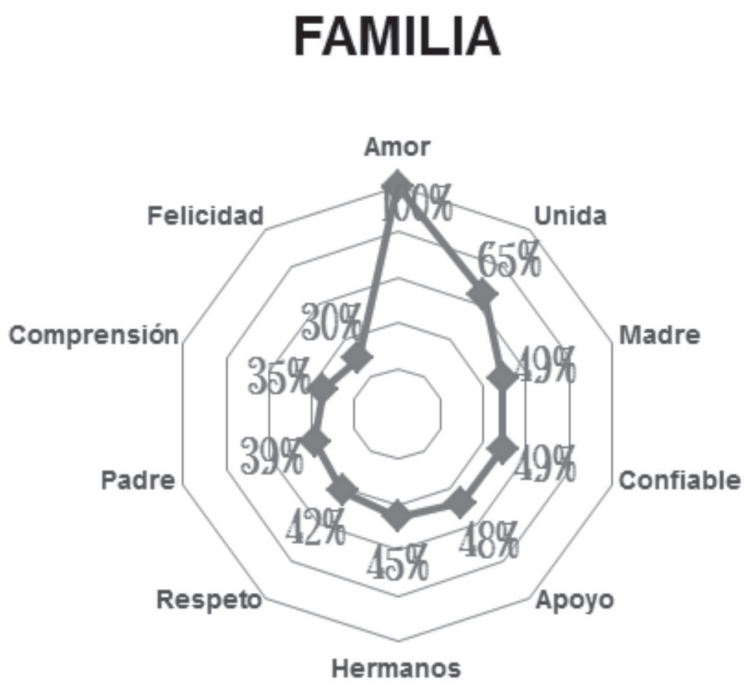

Figura 4.1. Distancia semántica cuantitativa del estímulo papá en la adolescencia tardía

\section{PAPÁ}

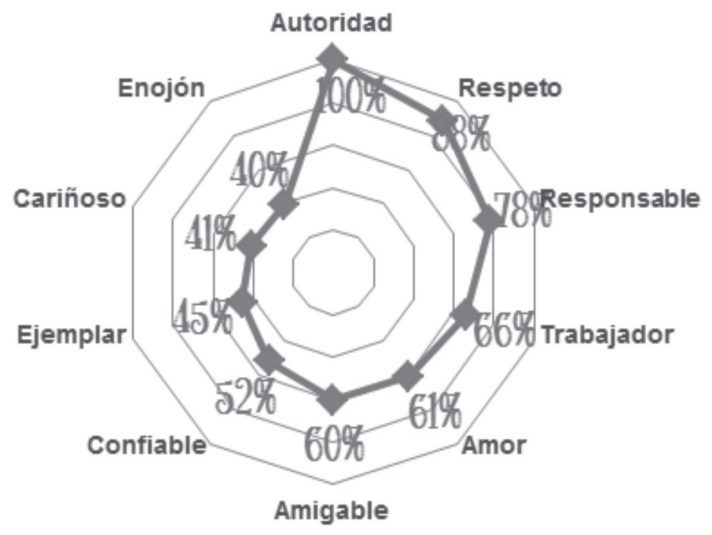

Figura 4.2. Distancia semántica cuantitativa del estímulo mamá en la adolescencia tardía

\section{MAMÁ}

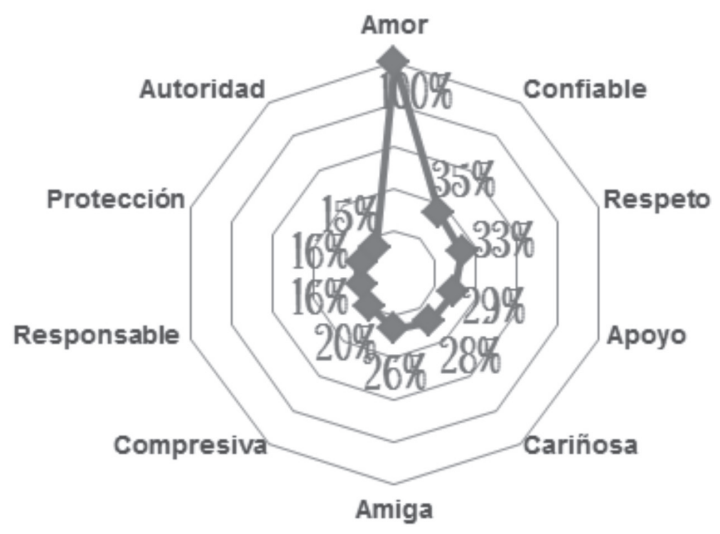




\section{Postadolescencia}

Al igual que para los grupos anteriores, se calculó el valor J de cada palabra estímulo de las redes generadas por los postadolescentes; a partir de ello se obtuvieron los siguientes valores:

$$
\text { Familia=144 } \quad \text { Papá=146 } \quad \text { Mamá=141 }
$$

En relación con el conjunto SAM y valor FMG, se observa que el nodo central de la red semántica del estímulo familia es amor, con el 100\%, seguido de unión con $51 \%$ y apoyo con $41 \%$, lo que pone en evidencia que la distancia semántica cuantitativa entre estas últimas y la definidora amor es bastante amplia, por lo que es posible afirmar que el amor es el significado psicológico de familia más importante para los jóvenes que se encuentran en la adolescencia tardía.

En relación con el estímulo papá, se obtuvo que la palabra amor es el nodo central de la red semántica ( $\mathrm{FMG}=100 \%)$, seguido de respetuoso $(\mathrm{FMG}=70 \%)$ y trabajador ( $\mathrm{FMG}=54 \%$ ), y que existe una distancia semántica cuantitativa de $30 \%$ por encima, con respecto a la segunda definidora (ver figura 5.1). De la misma forma que este caso, se observa que el núcleo central de la red del estímulo mamá es amor con el 100\% del valor FMG, seguido de cariñosa con $41 \%$, y las palabras apoyo y amiga, ambas con $28 \%$ (figura 5.2).

Figura 5. Distancia semántica cuantitativa del estímulo familia en la postadolescencia

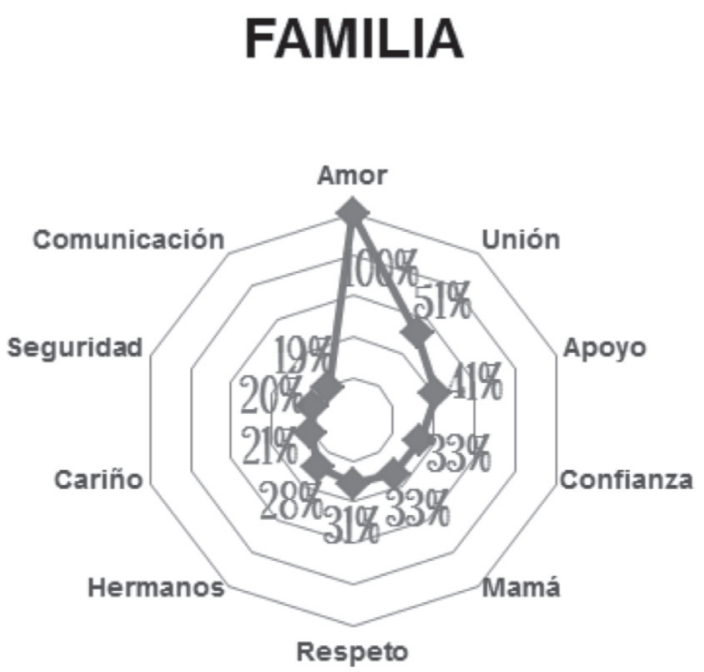

Figura 5.1. Distancia semántica cuantitativa del estímulo papá en la postadolescencia

\section{PAPÁ}

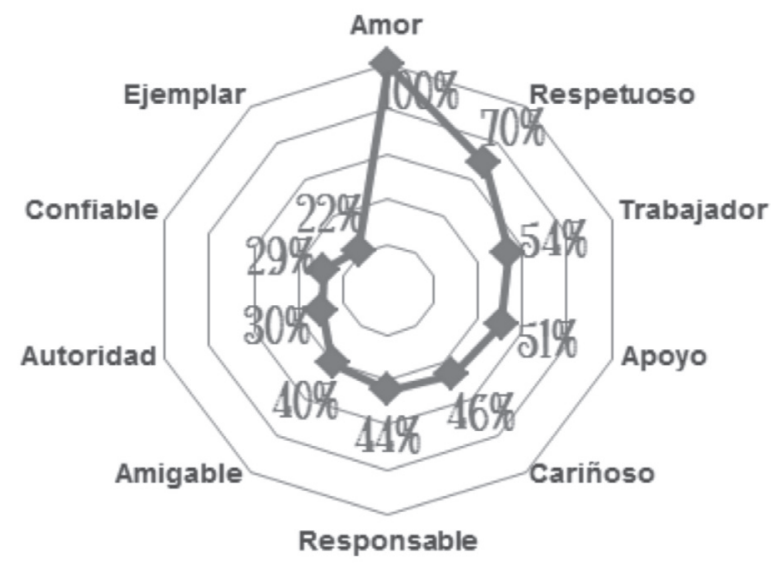

Figura 5.2. Distancia semántica cuantitativa del estímulo mamá en la postadolescencia

\section{MAMÁ}

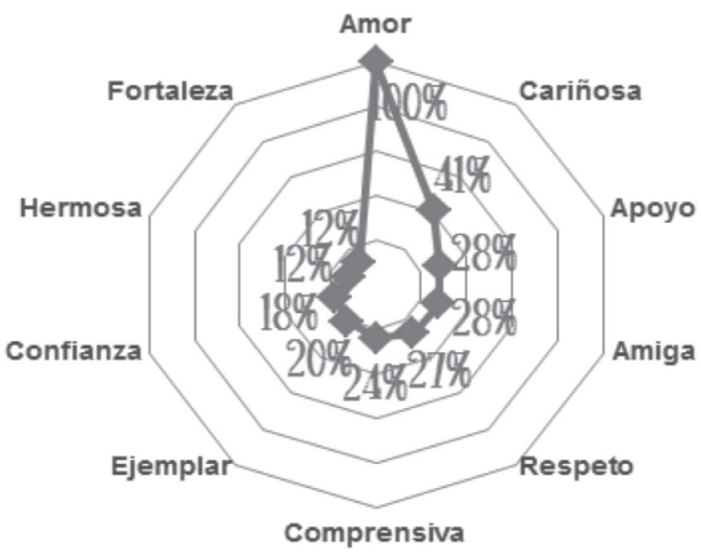




\section{DISCUSIÓN}

A partir de los análisis realizados con base en la técnica de Redes semánticas naturales se encontró que existen algunos elementos compartidos en las diferentes etapas de la adolescencia, ya que se otorga un significado a los estímulos familia, papá y mamá; sin embargo, se pudo observar que los conceptos estudiados poseen elementos claramente diferenciados en cada una de las subetapas de la adolescencia, tal como se evidenció en los resultados, en los que se demostró que los significados psicológicos varían conforme a la edad de los adolescentes, otorgando características muy particulares.

De acuerdo con el análisis realizado a partir de la comparación de los tamaños de red, en el caso de la palabra estímulo familia, se observó que la más amplia se generó en el grupo de preadolescentes, en tanto que la más pequeña fue en la adolescencia media. En cuanto al estímulo papá, el TR más amplio se dio en la adolescencia temprana, en tanto que la red más pequeña fue en la postadolescencia. De la misma manera, ante la palabra estímulo mamá, se observan características similares, ya que la red más amplia se generó en la adolescencia temprana, mientras que fue en la postadolescencia donde la red resultó más pequeña.

De acuerdo con Valdez (2002), el valor J es un indicador de la riqueza semántica de la red, es decir; a mayor cantidad de palabras, mayor riqueza de ésta. Sin embargo; Reyes Lagunes (1993), propone que el valor J se denomine simplemente "tamaño de la red" (TR), ya que no es claro que refleje dicho valor. Ante ello, se puede considerar que mientras más grande sea el tamaño de la red, el significado psicológico del concepto estudiado es poco claro y difuso, pues tal como se observó en los resultados, de manera general las redes más amplias, específicamente para las palabras estímulo familia, papá y mamá, fueron generadas por los grupos de preadolescentes y adolescentes tempranos, etapas que de acuerdo con Inhelder y Piaget (1996), corresponden a una capacidad cognitiva en pleno desarrollo, que aún carece de destreza necesaria para elaborar conceptos bien definidos.

Como característica general para todas las palabras estímulo se encontró que los tamaños de red disminuyeron considerablemente durante la adolescencia media. Al respecto, y desde las teorías de la adolescencia, Aberastury y Knobel (1971), plantean justa- mente que una de las formas típicas del pensamiento adolescente durante esta fase, corresponde al ensimismamiento, que le obliga a refugiarse y buscar respuestas en su interior, permitiendo un reajuste emocional que conlleva una preocupación por principios éticos, filosóficos y sociales, y le resta importancia a los problemas, luchas y rebeldías que fueron reflejo de los conflictos de la dependencia infantil. De esta manera equilibra sus conductas y emociones, otorgando un nuevo significado a la dimensión familiar, aunque aún con cierta dificultad para expresarlo, Con respecto a lo observado en cuanto a la distancia semántica cuantitativa; es decir, las palabras definidoras a las que se otorgaron los porcentajes más altos dentro del núcleo de la red, la familia como primera categoría de análisis fue vinculada en las primeras subetapas de la adolescencia (preadolescencia y adolescencia temprana), principalmente a elementos de la familia nuclear y extensa, mientras que a partir de la adolescencia media, así como en las etapas subsecuentes, fueron valores y emociones los que comenzaron a adquirir los lugares privilegiados al describir a la familia, haciendo alusión a conceptos que se adquieren en la misma como son el amor, la confianza, el apoyo y el respeto; y a su vez, integrando a su significado psicológico únicamente a los elementos que conforman la familia nuclear.

Coleman y Hendry (2003) plantean que durante la adolescencia ocurre un proceso de elaboración de conceptos que se consolida conforme transcurre el tiempo, donde el orden de aparición de estos conceptos se relaciona, en primer lugar, con el desarrollo intelectual durante la adolescencia, lo cual hace posible la elaboración de conceptos más complejos, que implican cada vez un mayor número de dimensiones, lo que permite incorporar además de las cualidades más observables, elementos intangibles como emociones y valores propios del sistema de creencias del adolescente.

Por otra parte, tanto preadolescentes como adolescentes tempranos asociaron al estímulo papá, cualidades observables y evaluaciones realizadas por los jóvenes, tales como trabajador, enojón, bueno, alegre, regañón y amigable; en contraste con lo que se observó en el grupo de la adolescencia media, donde los primeros lugares de la distancia semántica cuantitativa son ocupados por elementos que reflejan valores y emociones, en los que el amor y el cariño comienzan 
a adquirir un lugar importante dentro del núcleo de la red. Asimismo, se observó que en las etapas posteriores, las palabras que poseen el mayor peso semántico son indicadores de que los jóvenes perciben a su padre como una figura ejemplar, lo cual se refleja por medio del uso de conceptos tales como autoridad, respeto, responsable, trabajador y admiración; sin embargo, no es hasta la postadolescencia donde la palabra amor ocupa el significado psicológico de mayor relevancia, seguido de cualidades que indican que la relación entre padre e hijo se ve radicalmente fortalecida.

En la teoría, esto se ha asociado a diversos cambios que se producen en los adolescentes, como el incremento del pensamiento idealista con respecto a los padres y el incumplimiento de las expectativas y demandas; lo que genera conflictos entre padres e hijos adolescentes, mismos que disminuyen entre la adolescencia temprana y tardía (Laursen, Coy y Collins, 1998, en Santrock, 2004). Al respecto, González (2001) plantea que durante la adolescencia temprana aparece en los jóvenes una imperiosa necesidad de separarse de sus padres para continuar con su propio desarrollo, lo cual puede incitar la aparición de conflictos entre padres e hijos.

A diferencia del estímulo papá, el conflicto entre padres e hijos adolescentes del que se habla parece no ser tan evidente en el caso del estímulo mamá, resultando sumamente relevante como es que a partir de la adolescencia temprana, la palabra que muestra la mayor distancia semántica cuantitativa para dicho estímulo es amor, y siendo características positivas las que ocupan los lugares más altos dentro del núcleo de la red, principalmente a partir de la adolescencia media. Ante ello, Coleman y Hendry (2003) afirman que existen diferencias entre padres y madres al relacionarse con sus hijos adolescentes, pues se ha observado que ellas proporcionan mayor apoyo y están más interesadas y comprometidas en la tarea de educar a sus hijos; asimismo, mantienen un contacto íntimo con ellos y se comprometen en los intereses de los adolescentes, en tanto que los padres se caracterizan por fijar las metas a largo plazo y por proporcionar la disciplina. Aunado a ello, es necesario considerar además, que en gran parte de las culturas actuales se mantiene vigente un modelo de familia en el que la responsabilidad de quedarse en casa para brindar cuidado y educación a los hijos reside en la figura materna.
A manera de conclusión, merece destacar la importancia que los adolescentes otorgan a la familia, independientemente de la subetapa en que se encuentren. Los datos indican que, en general, se encuentran satisfechos con sus relaciones familiares y que identifican cualidades muy particulares para cada uno de los elementos que la integran, reconociendo el valor que cada uno aporta a la misma.

Para el adolescente, la familia es un espacio donde encuentra no sólo comprensión y apoyo, sino que también en ésta se consolidan los principios sobre los que edificará su identidad, a la par del devenir de cada subetapa de la adolescencia. En este sentido, los adolescentes logran ir integrando nuevos elementos al significado psicológico que tienen de sus padres y familia, manteniendo características muy similares en la construcción de los mismos, pues tal como se evidenció en los hallazgos, inicialmente aparecen características tangibles, a las cuales se van incorporando poco a poco diversos elementos, para concluir esta fase del desarrollo humano con un significado psicológico de familia, mamá y papá bien consolidado, que incorpora valores tanto familiares y personales, cualidades, sentimientos y emociones, los cuales dan cuenta de la importancia de fortalecer los lazos familiares durante esta etapa, para el desarrollo favorable del adolescente. 


\section{REFERENCIAS}

Aberastury, A. \& Knobel, M. (1971). La adolescencia normal, un enfoque psicoanalítico. Buenos Aires: Paidós.

Coleman, J. C. \& Hendry, L. (2003). Psicología de la adolescencia. Madrid: Morata.

Estrada, L. (2007). El ciclo vital de la familia. México: Random House Mondadori.

Gil Estallo, M. (2007). La familia: claves para una correcta gestión de las personas y situaciones familiares. Barcelona: Amat.

González, J. D. (2001). Psicopatología de la adolescencia. México: Manual Moderno.

Inhelder, B. \& Piaget, J. (1996). De la lógica del niño a la lógica del adolescente: Ensayo sobre la construcción de las estructuras operatorias formales. Barcelona: Paidós.

Roche Olivar, R. (2006). Psicología de la pareja y de la familia: Análisis y optimización. Barcelona: Servei de Publicacions Universitat Autónoma de Barcelona.
Santrock, J. (2004). Adolescencia. Madrid: McGraw Hill.

Valdez, J. L. (1991). Las categorías semánticas, usos y aplicaciones en psicología social. (Tesis de maestría inédita). UNAM: México.

Valdez, J. L. (2002). Las redes semánticas naturales, usos y aplicaciones en la psicología social. Toluca: Universidad Autónoma del Estado de México.

Zermeño, A. I., Arellano, A. C. \& Ramírez, V. A. (2005). Redes semánticas naturales: Técnica para representar los significados que los jóvenes tienen sobre televisión, internet y expectativas de vida. (Tesis de licenciatura). Universidad de Colima: Colima.

\section{NotA DEL AUTOR}

Esta investigación fue realizada en la Facultad de Psicología de la U.M.S.N.H., bajo la asesoría de la Mtra. Dámaris Díaz Barajas. 